\title{
SOCS4 expressed by recombinant HSV protects against cytokine storm in a mouse model
}

\author{
SHUQI REN ${ }^{1}$, XIAOQING CHEN ${ }^{2}$, RONGQUAN HUANG ${ }^{3}$, GRACE GUOYING ZHOU ${ }^{2,4}$ and ZHUQING YUAN ${ }^{1}$ \\ ${ }^{1}$ Department of Microbiology and Immunology, Guangzhou Medical University, Guangzhou, Guangdong 510182; \\ ${ }^{2}$ Immvira Co., Ltd., Shenzhen International Institute for Biomedical Research, Shenzhen, Guangdong 518116; \\ ${ }^{3}$ Department of Pathology; ${ }^{4}$ School of Basic Medical Sciences, Guangzhou Medical University, \\ Guangzhou, Guangdong 510182, P.R. China
}

Received June 20, 2018; Accepted November 30, 2018

DOI: $10.3892 /$ or.2018.6935

\begin{abstract}
Oncolytic viruses are genetically engineered viruses designed for the treatment of solid tumors, and are often coupled with the antitumor immunity of the host. The challenge of using oncolytic herpes simplex virus (oHSV) as an efficacious oncolytic agent is the potential host tissue damage caused by the production of a range of cytokines following intratumoral oHSV injection. An HSV-suppressor of cytokine signaling 4 (SOCS4) recombinant virus was created to investigate whether it inhibits cytokine storm. Recombinant HSV-SOCS4 and HSV-1(F) were used to infect mice, and levels of several representative cytokines, including monocyte chemoattractant protein-1, interleukin (IL)-1 $\beta$, tumor necrosis factor- $\alpha$, IL-6 and interferon $\gamma$, in serum and bronchoalveolar lavage fluid (BALF) of infected mice were determined, and immune cells in BALF and spleen were enumerated. Lung damage, virus titers in the lung, body weight and survival rates of infected mice were also determined and compared between the two groups. The cytokine concentration of HSV-SOCS4-infected mice was significantly decreased compared with that of HSV-1(F)-infected mice in BALF and serum, and a smaller number of cluster of differentiation $(C D) 11 b^{+}$cells of BALF, and $\mathrm{CD} 8{ }^{+} \mathrm{CD} 62 \mathrm{~L}^{+} \mathrm{T}$ cells and $\mathrm{CD} 4{ }^{+} \mathrm{CD} 62 \mathrm{~L}^{+} \mathrm{T}$ cells of the spleen were also identified in HSV-SOCS4-infected mice. HSV-SOCS4-infected mice exhibited slight lung damage, a decrease in body weight loss and a $100 \%$ survival rate. The results of the present study indicated that SOCS4 protein may
\end{abstract}

Correspondence to: Professor Grace Guoying Zhou, Immvira Co. Ltd., Shenzhen International Institute for Biomedical Research, 140 Jinye Avenue, Shenzhen, Guangdong 518116, P.R. China E-mail: zhoug@siitm.org.cn

Dr Zhuqing Yuan, Department of Microbiology and Immunology, Guangzhou Medical University, 101 XiuFa Avenue, Guangzhou, Guangdong 510182, P.R. China

E-mail: 2009990009@gzhmu.edu.cn

Key words: suppressor of cytokine signaling 4, herpes simplex virus, cytokine storm be a useful regulator to inhibit cytokine overproduction, and that HSV-SOCS4 may provide a possible solution to control cytokine storm and its consequences following induction by oncolytic virus treatment.

\section{Introduction}

Oncolytic viruses (OVs) are genetically engineered viruses that selectively replicate in and kill cancer cells, and represent a novel type of antitumor therapy (1-4). This approach has numerous advantages as a cancer therapeutic agent due to its mechanism-based selectivity, potential for mediating tumor cell death and possibility of expressing additional therapeutic transgenes at the tumor site $(5,6)$. Since OVs are designed for intratumoral injection, this unique cancer therapeutic is often coupled with the antitumor immunity (immunovirotherapy) of the host. The herpes simplex virus type 1 (HSV-1)-based oncolytic HSV (oHSV) talimogene laherparepvec (T-VEC, Imlygic) was the first US Food and Drug Administration-approved OV (7). As with other OV candidates, the host response against oHSV is complex, multifaceted, and modulated by host immunity and the tumor microenvironment. These various immune and inflammatory responses may be beneficial and detrimental $(8,9)$, among which, the induction of cytokine storm by $\mathrm{OV}$ delivery to particular organs (such as lung) is one of the increasingly recognized impediments. The term cytokine storm was first used to describe influenza-induced cytokine overproduction in a short time, and is associated with uncontrolled pro-inflammatory responses and significant immunopathology and severe disease outcomes (10). The development of cytokine storm with attendant pulmonary damage has subsequently been reported in various viral, bacterial and fungal infections (11). Consistent observations indicated that the concept of cytokine storm was much more complicated; however, our understanding of the mechanisms that promote cytokine storm remains limited, and countermeasures to control the balance between appropriate cytokine release and cytokine overproduction are relatively unexplored. The challenge of oHSV as an efficacious oncolytic agent is the potential tissue damage that is induced by a range of cytokine production following oHSV infection. Furthermore, the weakened immunity and unbalanced immune homeostasis of 
patients with tumors would exacerbate the situation. Therefore understanding the molecular mechanism of cytokine storm caused by oHSV injection and developing a strategy to control the hazards of cytokine storm following oHSV treatment become critically urgent.

The signaling cascade of a number of cytokines pass through the Janus kinase (JAK)/signal transducer and activator of transcription (STAT) pathway, and the suppressors of cytokine signaling (SOCS) proteins are pivotal negative regulators of the JAK/STAT signaling pathway that are part of cytokine networks (12). It has been identified that SOCS4-deficient mice exhibited increased lethality, which is associated with increased levels of pro-inflammatory cytokines in the lungs following influenza A virus infection (13). Accordingly, SOCS4 was postulated to be a critical regulator of antiviral immunity. However, very little is known regarding its function during oHSV infection.

In the present study, an HSV strain was constructed with a SOCS4 protein insert (HSV-SOCS4) to investigate its suppression of cytokine storm. The present study focused on several representative cytokines that serve key functions, including monocyte chemoattractant protein 1 (MCP-1), interleukin (IL)-1 $\beta$, tumor necrosis factor $\alpha$ (TNF- $\alpha$ ), IL-6 and interferon $\gamma$ (IFN- $\gamma$ ), and identified that their concentrations were decreased in HSV-SOCS4-infected mice compared with those in HSV-1(F)-infected mice, and HSV-SOCS4-infected mice exhibited slight lung damage, decreased weight loss and a $100 \%$ survival rate. The results of the present study indicate that HSV-SOCS4 may provide a possible solution to control the cytokine storm induced by oncolytic HSV therapy.

\section{Materials and methods}

Animals. A total of 60 female BALB/c mice, aged 6 weeks, were purchased from the Experiment Animal Center of Guangdong (Guangzhou, China) and were housed at $22 \pm 2^{\circ} \mathrm{C}$, ammonia $\leq 14$ ppm, 12-h light/12-h dark cycle, and free access to food and water, free from microbial pathogens at the Animal Center of Guangzhou Medical University (Guangzhou, China). All procedures involving mice were approved by the Institutional Animal Care and Use Committee of Guangzhou Medical University.

Cells and virus strains. Vero cells were obtained from the American Type Culture Collection (Manassas, VA, USA) and were cultured in Dulbecco's modified Eagle's medium (high glucose) supplemented with $5 \%(\mathrm{v} / \mathrm{v})$ fetal bovine serum or $5 \%$ (v/v) newborn calf serum (NBCS), respectively. All media were from HyClone; GE Healthcare (Logan, UT, USA). HSV-1(F), the prototype HSV-1 strain used in our laboratory (14), was propagated and titrated on Vero cells. pReceiver-M02 with human SOCS4 mRNA was purchased from GeneCopoeia, Inc. (Rockville, MD, USA).

Construction of HSV recombinant virus with SOCS4 expression. Two oligonucleotide primers were designed according to the human SOCS4 gene sequence: Forward, 5'-GTCGACATGTGG TGGCGCCTGTGGTGGCTCTGCTGCTGTGGCCCATGGT GTGGGCCGCAGAAAATAATGAAAATATTAG-3' (with an $A c c$ I site and underlined signal peptide $\mathrm{Hmm} 38$ ); reverse,

\section{5'-GCGGCCGCCTAGTGATGGTGATGGTGATGGCAT}

TGCTGTTCTGGTGCATC-3' (with a NotI site and underlined His tag). The polymerase chain reaction (PCR) was performed in a total reaction volume of $50 \mu \mathrm{l}$ for 30 cycles consisting of a denaturation step at $95^{\circ} \mathrm{C}$ for $1 \mathrm{~min}$, a primer annealing step at $58^{\circ} \mathrm{C}$ for $30 \mathrm{sec}$ and a primer extension step at $72^{\circ} \mathrm{C}$ for 2 min with Taq DNA polymerase (Thermo Fisher Scientific, Inc., Waltham, MA, USA). The PCR product of SOCS4 was ligated into T-easy plasmid and then was transformed into XL-1blue cells (Tiangen Biotech Co., Ltd., Beijing, China) and sent to IGE Biotech Ltd. (Guangzhou, China) for sequencing confirmation. The confirmed SOCS4 gene was excised from the T-easy plasmid and ligated into the carrier pNEWUL backbone site at ClaI/AccI and NotI (Fig. 1A). Next, the sequence between $B g l \mathrm{II}$ and PacI (including UL3, UL4 and SOCS4) was cleaved from pNEWUL and cloned into plasmid pKO5.1 at the same sites (Fig. 1B). The recombinant plasmid was transformed into a bacterial artificial chromosome (BAC) and cultured on a Luria-Bertani (LB) plate with chloramphenicol and zeocin at $43^{\circ} \mathrm{C}$ overnight. Next, the bacterial colony was selected and enriched on an LB plate with chloramphenicol and sucrose at $30^{\circ} \mathrm{C}$ overnight to excise plasmid pKO5.1. Following identification by PCR amplification of the SOCS4 gene (as aforementioned) and agarose (1\%) gel electrophoresis with ethidium bromide for visualization under UV, and analysis using Image Lab software (version 2.1; MCM Design, Hercules, CA, USA), the positive recombinant $\mathrm{BAC}$ was cultured to proliferate and was transformed into Vero cells using Lipofectamine ${ }^{\circledR}$ LTX with PLUS Reagent (Thermo Fisher Scientific, Inc.), according to the manufacturer's protocol. The cells were incubated until a viral cytopathic effect was observed. Viral plaques were collected in $1 \mathrm{ml}$ milk and then underwent three freeze-thaw $\left(-80^{\circ} \mathrm{C}\right.$ for $10 \mathrm{~min}$ and $37^{\circ} \mathrm{C}$ for $2 \mathrm{~min}$ ) cycles to release virus. The virus was inoculated and cultured into Vero cells in $25-\mathrm{cm}^{2}$ flasks, and DNA was extracted for PCR for final confirmation (including the UL3, UL4 and SOCS4 genes). Oligonucleotide primers for UL3 were 5'-GGATAGCAGATGTGAGGAAGTC-3' (forward) and 5'-ATGACACAGGCGCTCGGCATCG-3' (reverse) and for UL4 were 5'-GTATACCACCACCGTCGACATT-3' (forward) and 5'-GCGTTAAGGGGTCCGTTGTGTT-3' (reverse). PCR of $U L 3$ and $U L 4$ was performed in a total reaction volume of $50 \mu \mathrm{l}$ for 30 cycles consisting of a denaturation step at $95^{\circ} \mathrm{C}$ for $1 \mathrm{~min}$, a primer annealing step at $55^{\circ} \mathrm{C}$ for $30 \mathrm{sec}$ and a primer extension step at $72^{\circ} \mathrm{C}$ for 2 min with Taq DNA polymerase (Thermo Fisher Scientific, Inc.), and PCR amplification of the SOCS4 gene was as aforementioned. All PCR products were identified with $1.0 \%$ agarose gel electrophoresis with ethidium bromide for visualization under UV and Image Lab software (version 2.1). Expression of SOCS4 protein was determined by western blot analysis. Briefly, the recombinant virus-infected Vero cells were collected and centrifuged at $150 \mathrm{x}$ g for $20 \mathrm{~min}$ at $4^{\circ} \mathrm{C}$. The cell pellet was resuspended in $200 \mu \mathrm{l}$ cell lysis buffer (cat. no. P0013; Beyotime Institute of Biotechnology, Haimen, China) for $30 \mathrm{~min}$ on ice, and then the supernatant was collected and proteins were quantified using a Bicinchoninic Acid kit (cat no. P0010; Beyotime Institute of Biotechnology), according to the manufacturer's protocol. Proteins $(60 \mu \mathrm{g} / \mathrm{lane})$ were separated by SDS-PAGE (10\% gel). Subsequently, proteins were transferred onto a polyvinylidene difluoride (PVDF) membrane (cat. no. ISEQ00010; EMD Millipore, Billerica, MA, USA). The PVDF membrane was incubated for $2 \mathrm{~h}$ at 
room temperature in blocking solution (5\% non-fat milk in PBS containing $0.05 \%$ Tween-20). Following washing, the PVDF membrane was incubated overnight at $4^{\circ} \mathrm{C}$ in solution [PBS containing $0.05 \%$ Tween-20 and $1.0 \%$ bovine serum albumin (BSA; Sigma-Aldrich; Merck KGaA, Darmstadt, Germany)] containing 1:1,000 diluted anti-His-tag antibody (cat. no. 66005; ProteinTech Group, Inc., Chicago, IL, USA). The PVDF membrane was washed and incubated for $1 \mathrm{~h}$ at room temperature in the aforementioned solution containing 1:10,000 diluted horseradish peroxidase-conjugated goat anti-mouse immunoglobulin G secondary antibody(cat. no. 31430; Thermo Fisher Scientific, Inc.). Following washing, the result was determined using an Enlight ${ }^{\mathrm{TM}}$ Western kit (cat. no. 29050; Engreen Biosystem Co., Ltd., Auckland, New Zealand), according to the manufacturer's protocol. The desired recombinant virus (named HSV-SOCS4) was multiplied by culture, collected and stored at $-80^{\circ} \mathrm{C}$ until further use.

Infection of mice. BALB/c mice were divided randomly into three groups: One group was infected with HSV-1(F), one group was infected with HSV-SOCS4 and one group was treated with PBS as mock infection. In detail, each mouse was lightly anaesthetized and then was infected via the intranasal route with $10^{6}$ plaque-forming units of $\mathrm{HSV}-1(\mathrm{~F})$ or HSV-SOCS4 in $30 \mu \mathrm{l}$ PBS or treated with $30 \mu 1$ PBS only. Following infection, the mice were weighed and monitored for morbidity and mortality every day for 12 days.

Serum and bronchoalveolar lavage fluid samples collection and double sandwich ELISA. Orbital blood from each mouse was collected on days 1, 3 and 7 following infection, and the serum was separated and then stored at $-20^{\circ} \mathrm{C}$ for further ELISA. Following bleeding, the mouse was sacrificed and the lung was flushed three times with $1 \mathrm{ml}$ PBS through a blunted needle inserted into the trachea to collect bronchoalveolar lavage fluid (BALF). The samples were centrifuged at $100 \mathrm{x} \mathrm{g}$ for $20 \mathrm{~min}$ at $4^{\circ} \mathrm{C}$, the supernatant was removed for ELISA, and the cells were collected for flow cytometric analysis. For the BALF and serum samples, levels of a panel of cytokines, including MCP-1 (cat. no. 1217392), IL-1 $\beta$ (cat. no. 1210122), IL-6 (cat. no. 1210602), TNF- $\alpha$ (cat. no. 1217202) and IFN- $\gamma$ (cat. no. 1210002), were determined using a double sandwich ELISA (Dakewe Biotech Co., Ltd., Shenzhen, China), and the concentration of each cytokine was determined relative to the standard curve, according to the manufacturer's protocol.

Cell isolation and flow cytometric analysis. Cells harvested from BALF were treated with Tris/ $\mathrm{NH}_{4} \mathrm{Cl}$ to lyse erythrocytes, washed twice and resuspended in ice-cold RPMI-1640 medium (Thermo Fisher Scientific, Inc.). The spleen from each sacrificed mouse was eviscerated and fully ground, and the tissue was rinsed through a sterile wire screen. Spleen cell suspensions were collected, red blood cells inside were lysed, and the remaining cells were washed and cell pellet was resuspended in RPMI-1640 medium containing 1\% NBCS. BALF cells and spleen cells were counted and adjusted to $2 \times 10^{6}$ cells $/ \mathrm{ml}$. The cells were washed and stained for the following surface markers: Allophycocyanin (APC)-cluster of differentiation (CD)4 [APC-conjugated anti-mouse CD4 monoclonal antibody (mAb), clone GK1.5; cat. no. 104412], fluorescein isothiocyanate (FITC)-CD8a (FITC-conjugated anti-mouse CD8 mAb, clone 53-6.7; cat. no. 100706) and phycoerythrin (PE)-CD62L (PE-conjugated anti-mouse CD62L mAb, clone MEL-14; cat. no. 104408) for spleen cells and Pacific Blue (PB)-CD11b (PB anti-mouse CD11b mAb, clone M1/70; cat. no. 101224) for BALF cells. All antibodies were purchased from BioLegend, Inc. (San Diego, CA, USA). Cells were stained with 1:20 diluted antibody at $37^{\circ} \mathrm{C}$ for $10 \mathrm{~min}$, and the stained cells were washed with PBS containing $1 \%$ BSA and resuspended in $2 \%$ formaldehyde in PBS for flow cytometric analysis using a CytoFLEX flow cytometer (Beckman Coulter, Inc., Brea, CA, USA) and CytExpert software (version 2.0; Beckman Coulter, Inc.).

Lung samples for viral titration analysis and pathological analysis. Following bleeding and sacrifice, the lungs of two mice from each group were directly removed and minced completely with cell culture medium; the tissue homogenate was collected and centrifuged at $100 \mathrm{x}$ g for $20 \mathrm{~min}$ at $4^{\circ} \mathrm{C}$, and the supernatant was collected for viral titration analysis. Vero cells were cultured on 6 -well plates at $2 \times 10^{5}$ cells/well until $90 \%$ of cells formed a monolayer, and the supernatant sample was added. After $24 \mathrm{~h}$ of incubation at $37^{\circ} \mathrm{C}$, cells were removed carefully, centrifuged as aforementioned and the cell pellet was resuspended in $1 \mathrm{ml} \mathrm{milk}$, and stored at $-80^{\circ} \mathrm{C}$. Following three freeze-thaw cycles as aforementioned, the released virus sample was diluted 1:100, 1:1,000 and 1:10,000 with $1 \%$ NBCS. Next, $100 \mu \mathrm{l}$ each dilution was added to Vero cell monolayers on 6 -well plates followed by culture for $2 \mathrm{~h}$, the medium was replaced with basal medium Eagle (Thermo Fisher Scientific, Inc.) containing $1 \%$ NBCS and $0.5 \%$ immunoglobulin G (cat. no. S20013001; Taibang Biological Products Co, Ltd., Shandong, China), and the cells were incubated for an additional $72 \mathrm{~h}$. Subsequently, the cells were collected and stained with $0.03 \%$ methylene blue to quantify the plaques.

Pathological analysis of the mouse lung $(n=2$, without BALF collection) from HSV-1(F)-infected and HSV-SOCS4-infected mice was performed by the Department of Pathology at Guangzhou Medical University. The results were observed using an Eclipse 80i confocal microscope (Nikon Corporation, Tokyo, Japan).

Statistical analysis. Statistical analyses were performed using analysis of variance and Tukey's honestly significant difference test using SPSS statistical software (version 16.0; SPSS, Inc., Chicago, IL, USA) for multiple comparisons. $\mathrm{P}<0.05$ was considered to indicate a statistically significant difference.

\section{Results}

Construction of HSV-SOCS4 recombinant virus. The SOCS4 gene was inserted into a BAC to construct the HSV-SOCS4. The SOCS4 gene PCR product and sequencing identification confirmed the recombination. Fig. 1C presents the PCR product of SOCS4 (1,397 bp) from pReceiver-M02, and the final PCR confirmation of the fragments of $U L 4$ (1,492 bp), UL3 (1,319 bp) and SOCS4 (1,397 bp) is presented in Fig. 1D. A specific band was observed for HSV-SOCS4, but not for HSV-1(F), using western blot analysis (Fig. 1E), suggesting that SOCS4 protein was expressed. 
A Backbone:pNEWUL
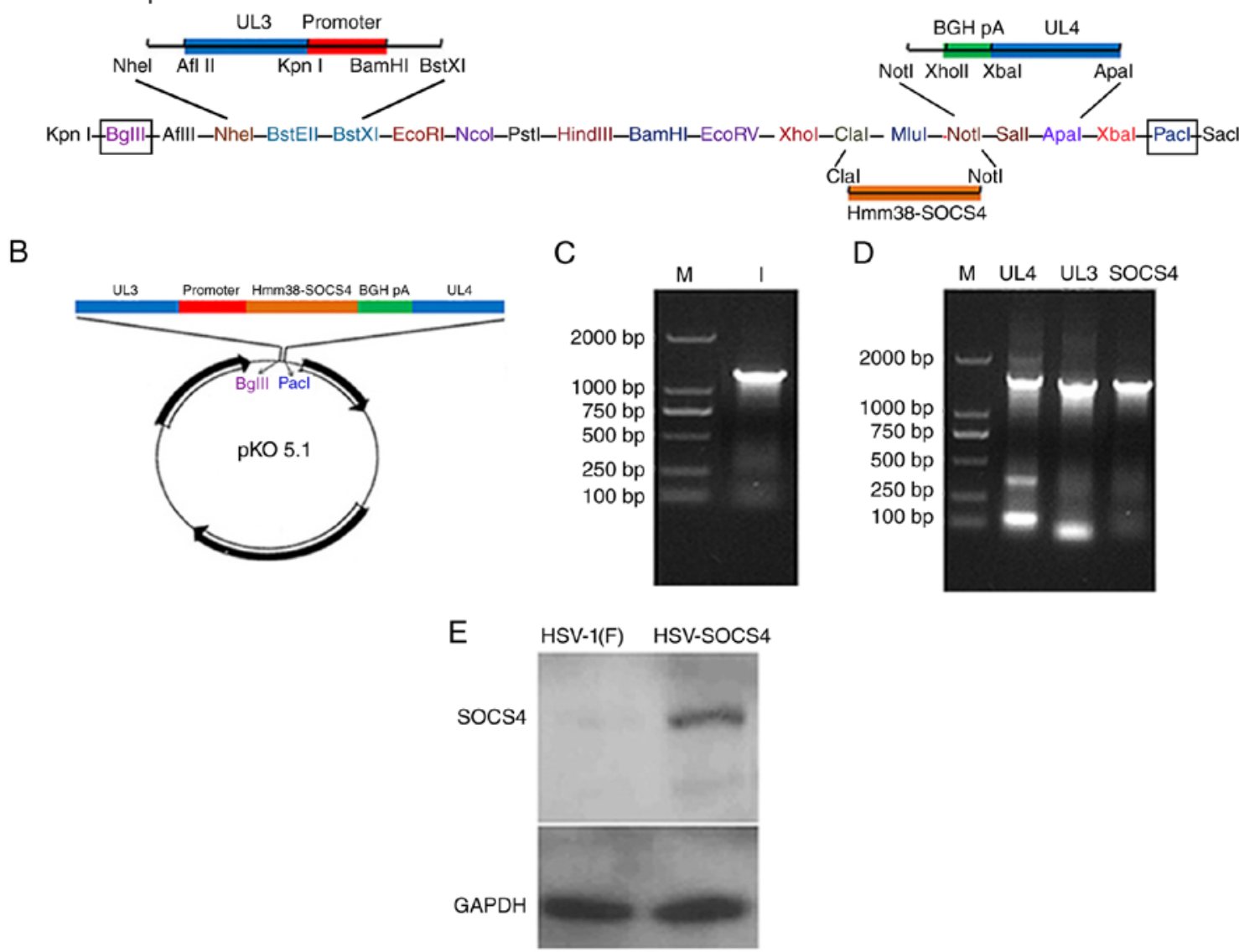

Figure 1. Schematic representation, PCR confirmation and western blot analysis of the HSV-SOCS4 recombinant. (A) The confirmed SOCS4 gene was ligated into the pNEWUL backbone at $C l a \mathrm{I} / A c c \mathrm{I}$ and $\operatorname{NotI}$ sites. (B) The sequence between BglII and PacI at the pNEWUL backbone (including $U L 3, U L 4$ and SOCS4) was cleaved from pNEWUL and cloned into plasmid pKO5.1 at the same site. (C) The band in lane 1 is the PCR product of SOCS4 (1,397 bp) from pReceiver-M02. (D) DNA was extracted from the reconstructed virus to perform PCR for the final confirmation, and bands between 1,000 and 2,000 bp were identified as: $U L 4$ (1,492 bp), UL3 (1,319 bp) and SOCS4 (1,397 bp). (E) A specific band of recombinant HSV-SOCS4 was observed, but not of HSV-1(F), from western blot analysis. PCR, polymerase chain reaction; HSV, herpes simplex virus; SOCS4, suppressor of cytokine signaling 4; M, molecular mass markers.

Cytokine production in BALF and serum following virus infection. To analyze the effect of SOCS4 protein on cytokine secretion, the profiles of the major inflammatory cytokines MCP-1, IL-1 $\beta$, IL-6, TNF- $\alpha$ and IFN- $\gamma$ were determined in BALF and serum from mice infected with PBS or HSV-1(F) or HSV-SOCS4 on days 1, 3 and 7 post-infection. Mock-infected mice did not induce appreciable amounts of cytokines in either BALF or serum at any time point investigated. The outcomes of cytokine production in BALF samples are presented in Fig. 2. A significantly increased level of all five cytokines was observed in HSV-1(F)-infected mice compared with that in HSV-SOCS4-infected mice on days 1, 3 and 7, except for IL-1 $\beta$ production on day 7 for which a negligible difference between the two groups of mice was observed. IL-1 $\beta$ production increased on day 3 compared with day 1 in HSV-1(F)-infected mice, but decreased by $\sim 50 \%$ on day 7 (Fig. 2B). IL-6 and IFN- $\gamma$ production in HSV-1(F)-infected mice was increased, but the greatest increase in IL-6 was observed on day 7, whereas that of IFN- $\gamma$ was observed on day 3 . The maximum level of MCP-1 in HSV-1(F)-infected mice was detected on day 1 and day 3 , but levels had decreased by day 7 . The highest TNF- $\alpha$ level was also observed on day 1 , but it had decreased when determined on day 3. The cytokine secretion of BALF from HSV-SOCS4-infected mice was consistent at the time points investigated with a slight difference, except for a significant increase in IL- 6 observed on day 7.

To determine the effect of SOCS4 protein on cytokine production in systemic circulation, mouse sera were collected for analysis by ELISA, and results are presented in Fig. 3. A significantly increased concentration of MCP-1 was detected in HSV-1(F)-infected mice compared with that in HSV-SOCS4-infected mice on day 1, and MCP-1 production in HSV-1(F)-infected mice decreased significantly at each time point, but was significantly decreased only on day 7 in HSV-SOCS4-infected mice. Additionally, the MCP-1 level in BALF was markedly higher compared with that in serum at every day measured in the two groups. IL-1 $\beta$ levels in HSV-1(F)- and HSV-SOCS4-infected mice were similar on day 1 and 3, but a significant increase was observed on day 7 in HSV-1(F)-infected mice. For TNF- $\alpha$ production, levels in HSV-SOCS4 were consistent over time, but TNF- $\alpha$ levels in HSV-1(F)-infected mice were increased compared with those in HSV-SOCS4-infected mice on days 1 and 3. The level of IL-6 was significantly increased in HSV-1(F)-infected mice over time, but a significantly increased level in HSV-SOCS4-infected mice was observed only on day 7. Additionally, the IL-6 level in HSV-1(F)-infected mice was significantly increased compared with in HSV-SOCS4-infected mice on every day 

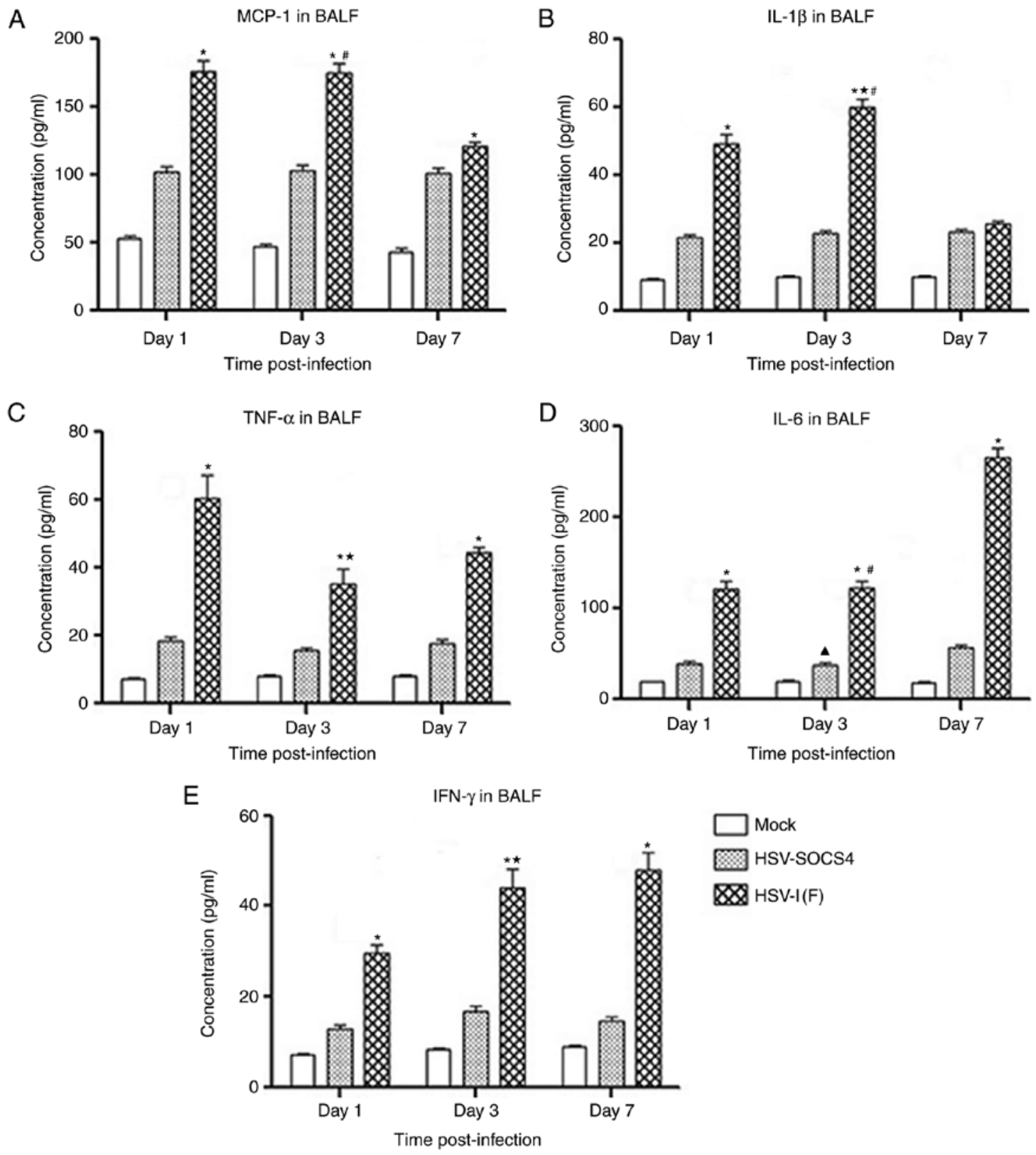

Figure 2. Cytokine production in BALF from mice infected with PBS (Mock), HSV-1(F) or HSV-SOCS4. BALF from each mouse (n=6) was collected on days 1, 3 and 7 following infection for the determination of cytokine levels by ELISA. (A) MCP-1 levels in BALF. (B) IL-1 $\beta$ levels in BALF. (C) TNF- $\alpha$ levels in BALF. (D) IL-6 levels in BALF. (E) IFN- $\gamma$ levels in BALF. ${ }^{\mathrm{P}}<0.05$ vs. HSV-SOCS4 on the same day; ${ }^{\star} \mathrm{P}<0.05$ vs. HSV-1(F) on day $1 ;{ }^{*} \mathrm{P}<0.05$ vs. HSV-1(F) on day 7; ${ }^{\wedge} \mathrm{P}<0.05$ vs. HSV-SOCS4 on day 7. BALF, bronchoalveolar lavage fluid; HSV, herpes simplex virus; SOCS4, suppressor of cytokine signaling 4; MCP-1, monocyte chemoattractant protein 1; IL, interleukin; TNF- $\alpha$, tumor necrosis factor $\alpha$; IFN- $\gamma$, interferon $\gamma$.

analyzed. For IFN- $\gamma$, the increases on day 1 and 3 were slight, but were significantly by day 7 in HSV-1(F)-infected mice and HSV-SOCS4-infected mice. The concentration of IFN- $\gamma$ in serum was increased compared with that in BALF on day 7 , confirming that activated T cells became the primary source of IFN- $\gamma$ production. To further determine cytokine production in serum of HSV-SOCS4-infected mice, cytokine levels were determined on day 12 , and no notable differences were observed (data not shown). In summary, the cytokine levels in BALF of HSV-SOCS4-infected mice were more consistent compared with those in serum.

Cell analysis of BALF and spleen following virus infection. To determine whether the diverse cytokine levels in BALF and serum were associated with the quantity of immune cells, cells from BALF and spleen of infected mice were collected for flow cytometric analysis. Since the difference in cytokine production was evident on days 1 and 7, cells were collected on these days to make the comparison. Considering that $\mathrm{CD} 1 \mathrm{~b}^{+}$cells, including macrophages, neutrophils and natural killer (NK) cells, constitute the main cell population present in BALF, the variation in $\mathrm{CD}_{1} 1 \mathrm{~b}^{+}$cells was quantified between HSV-1(F)- and HSV-SOCS4-infected mice. It was observed

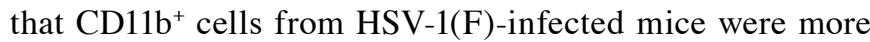
prevalent compared with those from HSV-SOCS4-infected mice, and there was a significant decrease in positively stained cells on day 7 compared with on day 1 in the two groups of infected mice (Fig. 4). 

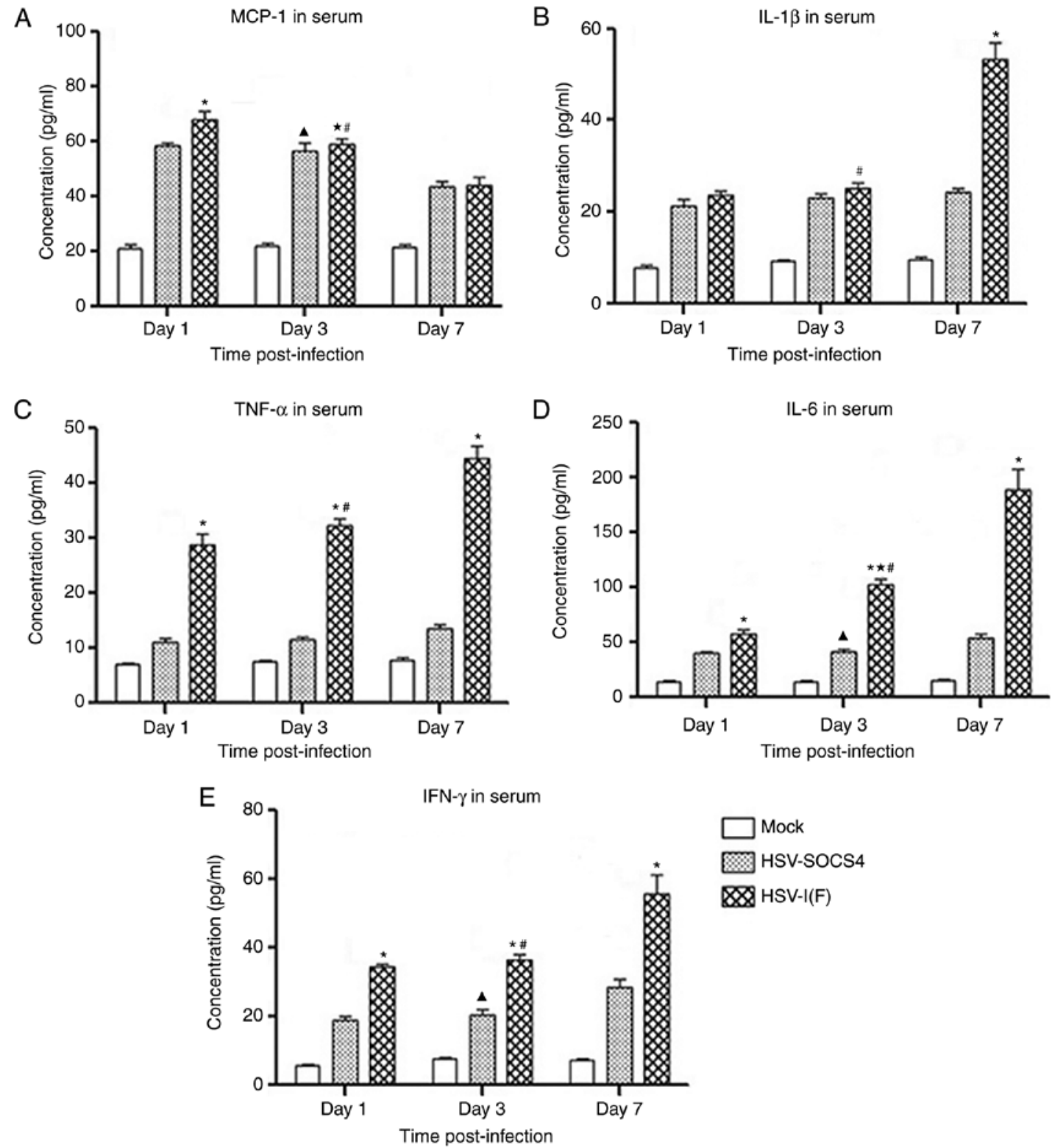

Figure 3. Cytokine production in serum from mice infected with PBS (Mock), HSV-1(F) or HSV-SOCS4. Serum from each mouse (n=6) was collected on days 1, 3 and 7 following infection for the determination of cytokine levels by ELISA. (A) MCP-1 levels in serum. (B) IL-1 $\beta$ levels in serum. (C) TNF- $\alpha$ levels in serum. (D) IL-6 levels in serum. (E) IFN- $\gamma$ levels in serum. ${ }^{*} \mathrm{P}<0.05$ vs. HSV-SOCS4 on the same day; ${ }^{\star} \mathrm{P}<0.05$ vs. HSV-1(F) on day 1 ; ${ }^{\# P}<0.05$ vs. HSV-1(F) on day 7; ${ }^{\triangle} \mathrm{P}<0.05$ vs. HSV-SOCS4 on day 7. HSV, herpes simplex virus; SOCS4, suppressor of cytokine signaling 4; MCP-1, monocyte chemoattractant protein 1 ; IL, interleukin; TNF- $\alpha$, tumor necrosis factor $\alpha$; IFN- $\gamma$, interferon $\gamma$.

$\mathrm{CD}^{+}{ }^{+}$and $\mathrm{CD} 8^{+}$cells from spleen were stained and CD62L was used as an activated marker. As presented in Fig. 5A, few double-positive cells were evident on day 1 in either group of infected mice, but an increased number of $\mathrm{CD} 8{ }^{+} \mathrm{CD} 62 \mathrm{~L}^{+}$ $\mathrm{T}$ cells was observed on day 7 . These differences were determined to be significant (Fig. 5B). A significant difference in the number between HSV-1(F) and HSV-SOCS4-infected mice was also observed (Fig. 5B). A similar pattern was also observed for $\mathrm{CD}^{+}{ }^{+} \mathrm{CD} 62 \mathrm{~L}^{+} \mathrm{T}$ cells (Fig. $5 \mathrm{C}$ and D).

Virus titers and pathological changes in the lung following virus infection. To determine the association of cytokine production with virus replication/clearance, the virus titer from infected mouse lung was quantified. The maximum virus titer was observed on day 1 , and evidently decreased by day 3 , and no virus was detected on day 7. Furthermore, the virus titer was significantly different between HSV-1(F)-infected mice and HSV-SOCS4-infected mice on day 3 (Fig. 6A).

The lungs of mice with no BALF collection underwent histopathological analysis, and representative images (at x200 magnification) are presented in Fig. 6B. On day 1, the lung of HSV-SOCS4-infected mouse barely exhibited any pathological changes, but evident cell infiltration with slight dilatation and hyperemia of local capillary was exhibited in the HSV-1(F)-infected mouse lung. On day 7, infiltration of a number of immune cells and mild-to-moderate dilatation and hyperemia of the capillary were observed in HSV-SOCS4-infected mouse lung, but the architecture of the lung alveolar wall was undisrupted. Additionally, a severe pathological change appeared in HSV-1(F)-infected 
A

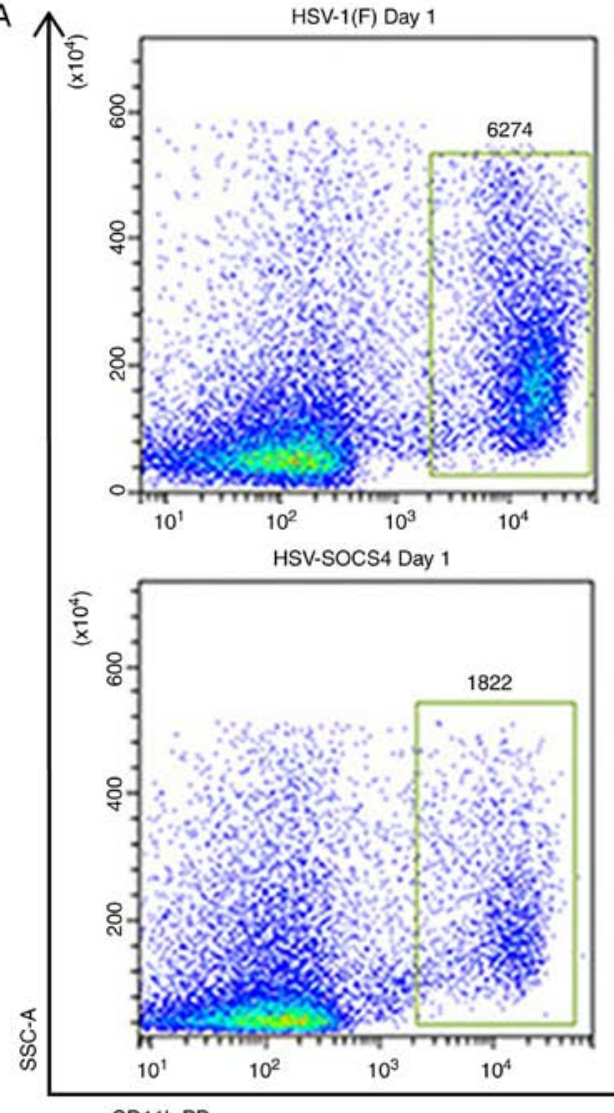

CD11b PB
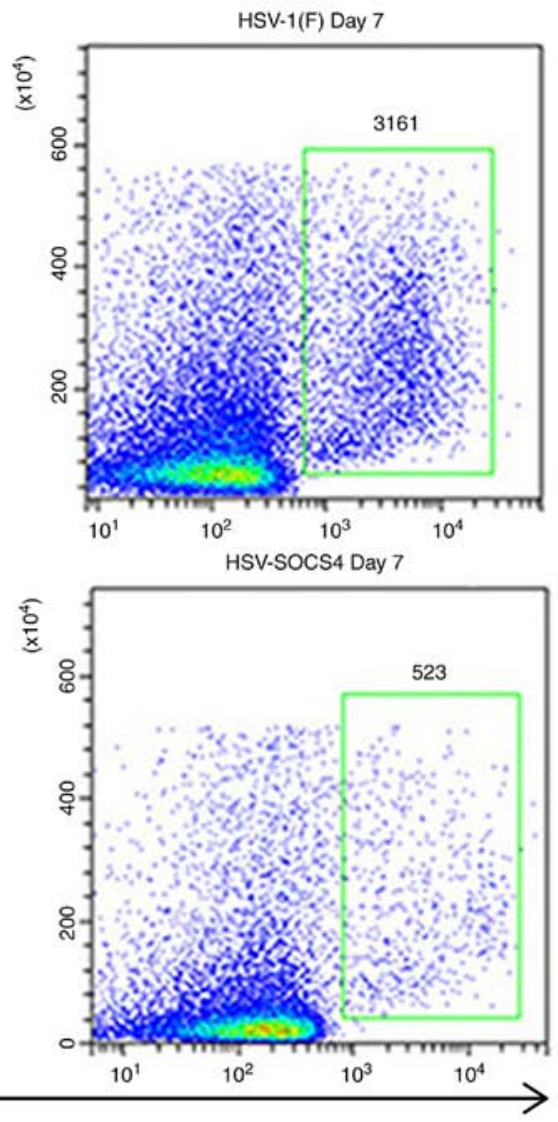

B

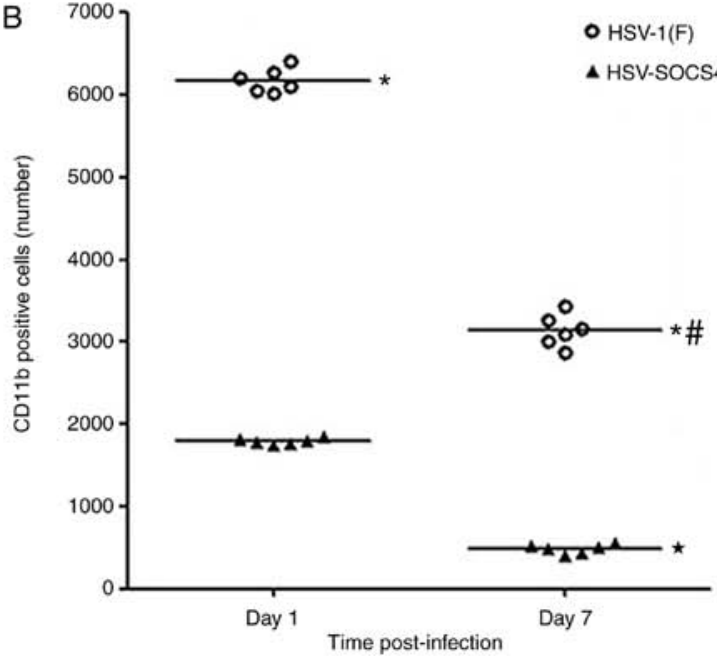

Figure 4. Flow cytometric analysis of BALF cells from mice infected with HSV-1(F) or HSV-SOCS4. BALF cells from mice were collected on days 1 and 7 following infection, stained with CD11b and analyzed by flow cytometry. (A) Representative plot from each group of mice on days 1 and 7 . The number of $\mathrm{CD}_{11 \mathrm{~b}}{ }^{+}$cells is indicated. (B) Quantification of results ( $\mathrm{n}=6$ mice). ${ }^{*} \mathrm{P}<0.05$ vs. HSV-SOCS4 on the same day; ${ }^{*} \mathrm{P}<0.05$ vs. HSV-1(F) on day 1 ; ${ }^{\star} \mathrm{P}<0.05$ vs. HSV-SOCS4 on day 1. BALF, bronchoalveolar lavage fluid; HSV, herpes simplex virus; SOCS4, suppressor of cytokine signaling 4; CD, cluster of differentiation; SSC, side scatter; PB, Pacific Blue.

mouse lung: A thickened and disrupted alveolar wall with severe surrounding hyperemia, accompanied by congested immune cells.

Body weight and mortality of mice following virus infection. Following infection, all mice were monitored twice daily for a period of 12 days to determine the body weight and onset of mortality. HSV-1(F)-infected mice started to lose their body weight gradually on day 2 , with a marked loss on day 7 , and the mice remaining alive had lost $50 \%$ of the body weight on day 10 (Fig. 7A). Consistently, the survival rate started to decline on day 7 (Fig. 7B), and then, the mice succumbed rapidly; no mouse from the HSV-1(F) group survived by day 11 . The mice in the HSV-SOCS4 group lost weight slightly and generally kept $80 \%$ of their weight on day 12 . The survival rate of HSV-SOCS4-infected mice was maintained at $100 \%$, which was significantly different from that of the HSV-1(F) group. Mock-infected mice exhibited no weight loss and no mortality. 

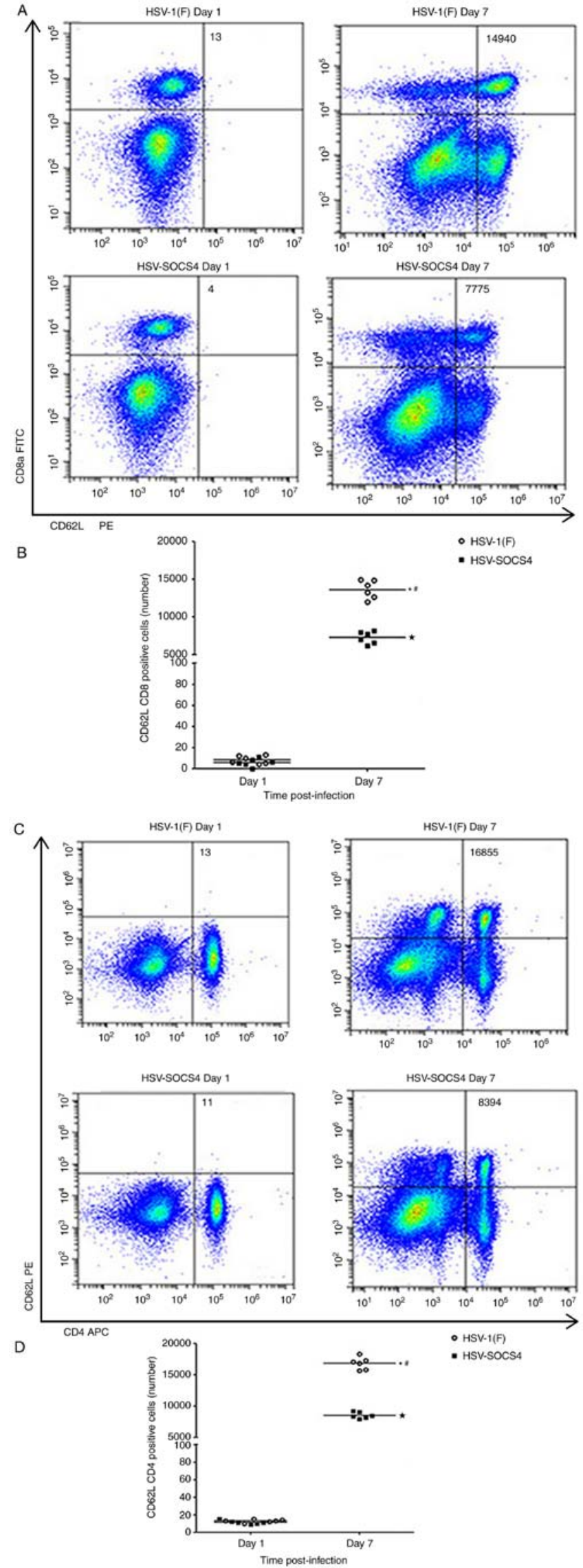

Figure 5. Flow cytometric analysis of spleen cells from mice infected with HSV-1(F) or HSV-SOCS4. Spleen cells from mice were collected on days 1 and 7 following infection, stained with CD62L, CD8a or CD4 and analyzed by flow cytometry. (A) Representative CD8 ${ }^{+} \mathrm{CD}_{2} \mathrm{~L}^{+} \mathrm{T}$ cell plot from each group of mice on days 1 and 7. The number of double positive cells is indicated. (B) Quantification of results ( $\mathrm{n}=6$ mice). (C) Representative $\mathrm{CD} 4{ }^{+} \mathrm{CD} 62 \mathrm{~L}^{+} \mathrm{T}$ cell plot from each group of mice on days 1 and 7. The number of double positive cells is indicated. (D) Quantification of results ( $\mathrm{n}=6 \mathrm{mice})$. ${ }^{*} \mathrm{P}<0.05 \mathrm{vs}$. HSV-SOCS4 on day $7 ;{ }^{*} \mathrm{P}<0.05$ vs. HSV-1(F) on day $1 ;{ }^{\star} \mathrm{P}<0.05$ vs. HSV-SOCS4 on day 1 . HSV, herpes simplex virus; SOCS4, suppressor of cytokine signaling 4; CD, cluster of differentiation; PE, phycoerythrin; APC, allophycocyanin. 
A

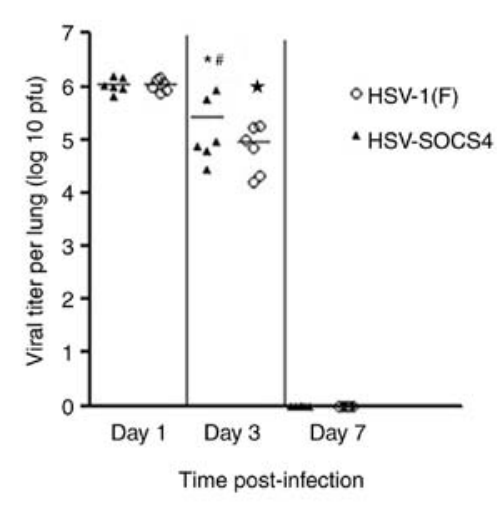

B

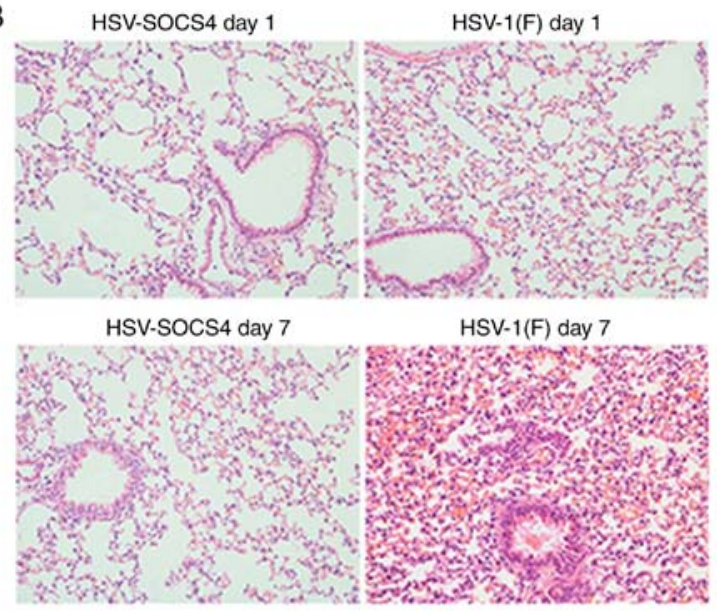

Figure 6. Viral titration and pathological analysis of infected mouse lungs. Following removal from the infected mice, the lungs were minced, and the supernatant was collected for viral titration analysis in Vero cell monolayers. (A) Viral titers on days 1, 3 and 7 following infection with HSV-1(F) or HSV-SOCS4 ${ }^{*} \mathrm{P}<0.05$ vs. HSV-SOCS4 on day $3 ;{ }^{*} \mathrm{P}<0.05$ vs. HSV-1(F) on day $1 ;{ }^{\star} \mathrm{P}<0.05$ vs. HSV-SOCS4 on day 1 . (B) Representative microscopic images at $\mathrm{x} 200$ magnification. HSV, herpes simplex virus; SOCS4, suppressor of cytokine signaling 4.
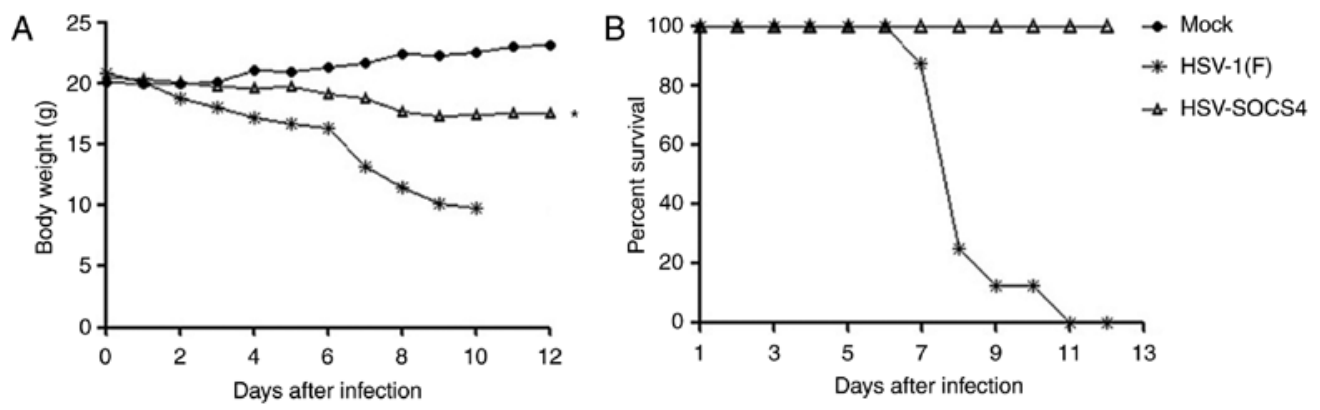

Figure 7. Body weight and mortality of mice following infection with PBS (Mock), HSV-1(F) or HSV-SOCS4. Following infection via the intranasal route, mice were monitored twice daily for a period of 12 days. (A) Average body weights (g) of all living mice in the three groups. ${ }^{*} \mathrm{P}<0.05 \mathrm{vs}$. HSV-1(F). (B) Survival rates of mice in the three groups.

\section{Discussion}

HSV-1 infects various mucosal tissues, including the respiratory tract, and it was identified previously that HSV-1-inducted pneumonia was due to the inflammatory response rather than direct cytopathic effects of the virus itself (15), indicating the potential risk of using oHSV as antitumor therapy, particularly because patients with cancer are generally in disordered immuno-homeostasis. This uncontrolled inflammatory response is the consequence of an excessive release of pro-inflammatory cytokines, known as cytokine storm. Unfortunately, the understanding of molecules involved in cytokine storm, contribution of cytokines to pathogenesis, and therapeutic strategies to prevent or alleviate symptoms remain insufficient. We hypothesized that increased negative feedback to the immune system or inhibiting the overproduction of inflammatory cytokines may be a prospective method to prevent tissue and organ damage caused by cytokine storm.

Unlike SOCS1, SOCS3 and other members of the SOCS family that have been well-described (16-18), studies concerning SOCS4 protein are relatively scarce. SOCS4 is known to be responsible for the inhibition of immune signaling by STAT3, which is associated with human cancer therapy $(19,20)$. Mice lacking functional SOCS4 were hypersusceptible to primary infection with influenza A virus, and displayed dysregulated pro-inflammatory cytokine production in the lungs, delayed viral clearance and impaired trafficking of influenza-specific $\mathrm{CD}^{+} \mathrm{T}$ cells to the site of infection (13). Therefore, SOCS4 may be a promising protein to control the excessive release of pro-inflammatory cytokines induced by oHSV treatment. To validate our hypothesis, a novel HSV-1 strain with an inserted SOCS4 gene named HSV-SOCS4 was constructed, and it was used to infect mice intranasally to evaluate whether cytokine storm could be inhibited.

Since cytokines instilled into the lungs could pass into the bloodstream, providing direct communication between local and systemic response, BALF and serum samples were collected for the determination of several cytokines that are involved in cytokine storm on days 1, 3 and 7 post-infection. Produced primarily by dendritic cells (DCs) and macrophages, IL-1 $\beta$ is a key cytokine driving pro-inflammatory activity. It promotes recovery when present early in infection, but is associated with a damaging inflammatory response, leading to severe pathogenesis and mortality when present at late stages of infection (21). It was identified that IL-1 $\beta$ remained at a high level at the early stages (days 1 and 3) in BALF samples and was degraded on day 7 . By contrast, its production in serum was increased on day 7 in HSV-1(F)-infected 
mice. BALF and serum IL-1 $\beta$ maintained corresponding low levels during all time points in HSV-SOCS4-infected mice. These data suggested that SOCS4 protein may inhibit early IL-1 $\beta$ production in BALF and later production in serum. IL-1 and IL-6 are synergistic, but IL-1 is expressed principally in the early stages of infection, followed by an increase in the expression of IL-6 (22). The production of IL-6 in BALF following HSV infection has been reported (23), and the results of the present study were consistent: Strong upregulation of IL-6 levels in BALF of HSV-1(F)-infected mice was evident, followed by a sustained increase, particularly on day 7; and similar results were also evident in serum with a relatively lower concentration. Restricted levels of IL-6 were apparent in BALF and serum in HSV-SOCS4-infected mice and exhibited an increase on day 7. The marked later production of IL-6 was independent of the presence of virus (24) and may be associated with promotion of Th2 responses (25). TNF- $\alpha$, another prominent acute-response cytokine, is primarily produced by macrophages, lung epithelial cells and helper $\mathrm{T}$ cells, and may appear at early times following infection. TNF- $\alpha$ contributes to the symptoms of severe disease following $\mathrm{H} 5 \mathrm{~N} 1$ virus infection and represents the quintessential features of cytokine storm $(26,27)$; it is also involved in immunopathology associated with HSV infection (28). TNF- $\alpha$ was released from the innate immune system through virus interaction with macrophages and NK cells at early times following infection (a notably high level of TNF- $\alpha$ was observed in BALF on day 1 in the present study), and the adaptive immune system via activation of virus-specific $\mathrm{CD} 4^{+}$or $\mathrm{CD}^{+} \mathrm{T}$ cells [an increased level of TNF- $\alpha$ was observed in serum on day 7 from HSV-1(F)-infected mice], but variations in the TNF- $\alpha$ level in HSV-SOCS4-infected mice were indistinguishable at every time point in BALF and serum samples. It was reported that anti-TNF- $\alpha$ treatment decreased the severity of weight loss and illness following $\mathrm{H} 3 \mathrm{~N} 2$ virus challenge, indicating that it may be a promising therapeutic target (29), and we hypothesized that restrained TNF- $\alpha$ production may also decrease the symptoms caused by HSV infection. MCP-1 is rapidly produced by various cell types, primarily monocytes, macrophages, epithelial cells and endothelial cells, following inflammatory stimuli and tissue damage. It recruits monocytes, memory T cells, NK cells and DCs to the sites of tissue injury and infection $(30,31)$. A markedly higher level of MCP-1 in BALF was observed at early stages from HSV-1(F)-infected mice, consistent with the observation that more $\mathrm{CD} 11 \mathrm{~b}^{+}$cells were present in HSV-1(F)-infected mouse BALF samples. IFN- $\gamma$ is a potent cytokine with numerous functions, including promoting the activation of DCs and macrophages, enhancing the cytotoxicity of NK cells and inducing antibody production of B cells (32). The increased IFN- $\gamma$ level of HSV-1(F)-infected mice on day 3 in BALF was produced primarily by NK cells at the early stage of infection and it helped to control viral replication (33); the increased level in serum from the two groups of infected mice at the later stage (day 7) was because T cells became the principal source of IFN- $\gamma$. Certain activities of IFN $-\gamma$ in the later response had been associated with inflammation and lung injury (34), possibly explaining the lung damage in HSV-1(F)-infected mice. Prolonged and indiscriminate IFN- $\gamma$ production was observed in the serum samples of HSV-SOCS4-infected mice on day 12 when the virus could not be identified in lung tissue, confirming that IFN- $\gamma$ could be induced downstream by other cytokines or features of the immune response (35), and SOCS4 protein may inhibit the prolonged production in serum.

Pro-inflammatory cytokines are responsible for cell activation and tissue damage; additionally, the release of one cytokine may induce new cytokine production, which will in turn further cause cell and organ necrosis. The consistently low level of pro-inflammatory cytokine production in BALF and serum of HSV-SOCS4-infected mice is thought to be associated with the amelioration of mice lung damage compared with that of HSV-1(F)-infected mice.

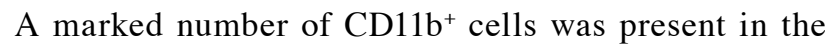
HSV-1(F)-infected mouse BALF on day 1 , which was the consequence of the high level of MCP-1 in BALF. It was reported that macrophages serve an essential function in the first line of defense against HSV within the lung by rapidly secreting a primary wave of pro-inflammatory cytokines (36), explaining the increased production of TNF- $\alpha$, IL-1 $\beta$ and IL- 6 in BALF of HSV-1(F)-infected mice on day 1, because macrophages and NK cells are the primary source of those cytokines in the initial response. Recruitment of macrophages into the lung and alveolar spaces is a hallmark of the initial immune response, and $\mathrm{CD}^{+}$and $\mathrm{CD} 8^{+} \mathrm{T}$ cells in the spleen may reflect the activity of the adaptive immune response. CD62L is generally used as an activation marker of $\mathrm{T}$ cells, and it serves a major function in directing lymphocytes to the site of infection and inflammation (37). No activated $T$ cells were identified in the spleen on day 1 from either group of infected mice, but marked activation of cells was evident on day 7 , and the $\mathrm{CD} 4{ }^{+} \mathrm{CD} 62 \mathrm{~L}^{+} \mathrm{T}$ cell number and the $\mathrm{CD} 8{ }^{+} \mathrm{CD} 62 \mathrm{~L}^{+} \mathrm{T}$ cell number in $\mathrm{HSV}-1(\mathrm{~F})$-infected mice were increased 2-fold compared with those of HSV-SOCS4-infected mice, a result that was associated with the increased level of TNF- $\alpha$, IL-1 $\beta$, IL- 6 and IFN- $\gamma$ in serum of HSV-1(F)-infected mice on day 7. Effective Th cells and CTLs are critical for the efficient resolution of viral infection through the production of cytokines and/or direct lysis of infected cells; however, these same mechanisms also contribute to pulmonary damage (38).

The virus titer in the lung was similar between HSV-1(F)and HSV-SOCS4-infected mice on day 1, indicating that the two groups of mice were equally infected. However, the levels declined on day 3 , and an evident difference was detected between the two groups. This rapid virus clearance was consistent with innate immunity mechanisms, and, due to the activation of a larger quantity of immune cells (as observed by flow cytometric analysis and pathological analysis on day 1), a decreased virus load was detected in HSV-1(F)-infected mouse lung. Typically, lytic infection is shut off on day 7; therefore, no virus was tested. Following HSV-1(F) infection, serious pathological changes in the mouse lung were observed on day 7 , consistent with the increased cytokine levels and $\mathrm{T}$ cells of the mice. Owing to lung damage (possibly involving other organ lesions), excessive weight loss of HSV-1(F)-infected mice started on day 7 , and the mortality rate reached $75 \%$ on day 8 and $100 \%$ on day 11 . Conversely, HSV-SOCS4-infected mice exhibited only slight weight loss and no mortality on day 12 . Those results indicated that controlling cytokine over-release 
could maintain the body weight, health and survival of infected mice. The results of the present study indicated that HSV with SOCS4 protein insertion inhibited cytokine overproduction, excessive infiltration of immune cells, alleviated lung pathological damage and decreased the mortality rate of mice.

Immunity to virus infection is multifaceted and highly complex, and cytokine induction is a series of sprawling network with redundancy and amplified cascades. Thus, intervention strategies should target multiple cytokine pathways. The attempt in the present study to create a recombinant HSV-1 strain with SOCS4 expression was to provide a valuable tool to suppress the cytokine storm and its disastrous consequence, which may improve oHSV clinical treatment. The molecular mechanisms of SOCS4 in the cytokine pathway and effects of the HSV-SOCS4 strain in mice with tumor and other animals requires further investigation.

\section{Acknowledgements}

Not applicable.

\section{Funding}

The present study was funded by the National Nature Science Foundation of China (grant no. NSFC 81472826), the Shenzhen Science and Innovation Commission Project (grant no. CYZZ20150831144745459), and the National Major Scientific and Technological Special Project for Significant New Drugs Development during the Thirteenth Five-year Plan Period (grant no. 2018ZX09733002).

\section{Availability of data and materials}

All data generated or analyzed during this study are included in this published article.

\section{Authors' contributions}

SR performed all sample collection, double sandwich ELISA, and flow cytometric analysis. XC recombined the HSV-SOCS4 and cell culture. RH performed the mice infection and pathological analysis of mouse lung. GGZ contributed to the study design and wrote the manuscript. ZY designed the study, analyzed the data and drafted the manuscript. All authors read and approved the final manuscript.

\section{Ethics approval and consent to participate}

The present study does not contain any studies with human participants performed by any of the authors. All procedures involving mice were approved by the Institutional Animal Care and Use Committee of Guangzhou Medical University.

\section{Patient consent for publication}

Not applicable.

\section{Competing interests}

The authors declare that they have no competing interests.

\section{References}

1. Russell SJ, Peng KW and Bell JC: Oncolytic virotherapy. Nat Biotechnol 30: 658-670, 2012.

2. Jhawar SR, Thandoni A, Bommareddy PK, Hassan S, Kohlhapp FJ, Goyal S, Schenkel JM, Silk AW and Zloza A: Oncolytic viruses-natural and genetically engineered cancer immunotherapies. Front Oncol 7: 202, 2017.

3. Maroun J, Muñoz-Alía M, Ammayappan A, Schulze A, Peng KW and Russell S: Designing and building oncolytic viruses. Future Virol 12: 193-213, 2017.

4. Lin CZ, Xiang GL, Zhu XH, Xiu LL, Sun JX and Zhang XY: Advances in the mechanisms of action of cancer-targeting oncolytic viruses. Oncol Lett 15: 4053-4060, 2018.

5. Kaufman HL, Kohlhapp FJ and Zloza A: Oncolytic viruses: A new class of immunotherapy drugs. Nat Rev Drug Discov 14: 642-662, 2015

6. Loskog A: Immunostimulatory gene therapy using oncolytic viruses as vehicles. Viruses 7: 5780-5791, 2015.

7. Peters $C$ and Rabkin SD: Designing herpes viruses as oncolytics. Mol Ther Oncolytics 2: pii: 15010, 2015.

8. Saha D, Wakimoto $\mathrm{H}$ and Rabkin SD: Oncolytic herpes simplex virus interactions with the host immune system. Curr Opin Virol 21: 26-34, 2016

9. Chaurasiya S, Chen NG and Fong Y: Oncolytic viruses and immunity. Curr Opin Immunol 51: 83-90, 2018.

10. Tisoncik JR, Korth MJ, Simmons CP, Farrar J, Martin TR and Katze MG: Into the eye of the cytokine storm. Microbiol Mol Biol Rev 76: 16-32, 2012.

11. Chousterman BG, Swirski FK and Weber GF: Cytokine storm and sepsis disease pathogenesis. Semin Immunopathol 39: 517-528, 2017.

12. Alexander WS: Suppressors of cytokine signalling (SOCS) in the immune system. Nat Rev Immunol 2: 410-416, 2002.

13. Kedzierski L, Linossi EM, Kolesnik TB, Day EB, Bird NL, Kile BT, Belz GT, Metcalf D, Nicola NA, Kedzierska K, et al: Suppressor of cytokine signaling 4 (SOCS4) protects against severe cytokine storm and enhances viral clearance during influenza infection. PLoS Pathog 10: e1004134, 2014.

14. Liu Y, Qu L, Liu Y, Roizman B and Zhou GG: PUM1 is a biphasic negative regulator of innate immunity genes by suppressing LGP2. Proc Natl Acad Sci USA 114: E6902-E6911, 2017.

15. Adler H, Beland JL, Del-Pan NC, Kobzik L, Brewer JP, Martin TR and Rimm IJ: Suppression of herpes simplex virus type 1 (HSV-1)-induced pneumonia in mice by inhibition of inducible nitric oxide synthase (iNOS, NOS2). J Exp Med 185: 1533-1540, 1997.

16. Liau NPD, Laktyushin A, Lucet IS, Murphy JM, Yao S, Whitlock E, Callaghan K, Nicola NA, Kershaw NJ and Babon JJ: The molecular basis of JAK/STAT inhibition by SOCS1. Nat Commun 9: 1558, 2018.

17. Mahony R, Ahmed S, Diskin C and Stevenson NJ: SOCS3 revisited: A broad regulator of disease, now ready for therapeutic use? Cell Mol Life Sci 73: 3323-3336, 2016.

18. Linossi EM, Babon JJ, Hilton DJ and Nicholson SE: Suppression of cytokine signaling: The SOCS perspective. Cytokine Growth Factor Rev 24: 241-248, 2013.

19. Trengove MC and Ward AC: SOCS proteins in development and disease. Am J Clin Exp Immunol 2: 1-29, 2013.

20. Wang X, Crowe PJ, Goldstein D and Yang JL: STAT3 inhibition, a novel approach to enhancing targeted therapy in human cancers (Review). Int J Oncol 41: 1181-1191, 2012.

21. Tate MD, Ong JD, Dowling JK, McAuley JL, Robertson AB, Latz E, Drummond GR, Cooper MA, Hertzog PJ and Mansell A: Reassessing the role of the NLRP3 inflammasome during pathogenic influenza A virus infection via temporal inhibition. Sci Rep 6: 27912, 2016.

22. Kaiser L, Fritz RS, Straus SE, Gubareva L and Hayden FG: Symptom pathogenesis during acute influenza: Interleukin- 6 and other cytokine responses. J Med Virol 64: 262-268, 2001.

23. Murphy EA, Davis JM, Brown AS, Carmichael MD, Ghaffar A and Mayer EP: Effect of IL-6 deficiency on susceptibility to HSV-1 respiratory infection and intrinsic macrophage antiviral resistance. J Interferon Cytokine Res 28: 589-595, 2008.

24. Tengku-Muhammad TS, Hughes TR, Ranki H, Cryer A and Ramji DP: Differential regulation of macrophage CCAAT-enhancer binding protein isoforms by lipopolysaccharide and cytokines. Cytokine 12: 1430-1436, 2000. 
25. Hunter CA and Jones SA: IL-6 as a keystone cytokine in health and disease. Nat Immunol 16: 448-457, 2015.

26. Peiris JS, Cheung CY, Leung CY and Nicholls JM: Innate immune responses to influenza A H5N1: Friend or foe? Trends Immunol 30: 574-584, 2009.

27. Perrone LA, Szretter KJ, Katz JM, Mizgerd JP and Tumpey TM Mice lacking both TNF and IL-1 receptors exhibit reduced lung inflammation and delay in onset of death following infection with a highly virulent H5N1 virus. J Infect Dis 202: 1161-1170, 2010.

28. Fujii S, Akaike T and Maeda H: Role of nitric oxide in pathogenesis of herpes simplex virus encephalitis in rats. Virology 256 203-212, 1999.

29. Hussell T, Pennycook A and Openshaw PJ: Inhibition of tumor necrosis factor reduces the severity of virus-specific lung immunopathology. Eur J Immunol 31: 2566-2573, 2001.

30. Deshmane SL, Kremlev S, Amini S and Sawaya BE: Monocyte chemoattractant protein-1 (MCP-1): An overview. J Interferon Cytokine Res 29: 313-326, 2009.

31. Rose CJ, Sung SS and Fu SM: Significant involvement of CCL2 (MCP-1) in inflammatory disorders of the lung. Microcirculation 10: 273-288, 2003.

32. Billiau A and Matthys P: Interferon-gamma: A historical perspective. Cytokine Growth Factor Rev 20: 97-113, 2009.
33. Reading PC, Whitney PG, Barr DP, Smyth MJ and Brooks AG: NK cells contribute to the early clearance of HSV-1 from the lung but cannot control replication in the central nervous system following intranasal infection. Eur J Immunol 36: 897-905, 2006.

34. Ramana CV, DeBerge MP, Kumar A, Alia CS, Durbin JE and Enelow RI: Inflammatory impact of IFN- $\gamma$ in $\mathrm{CD}^{+} \mathrm{T}$ cell-mediated lung injury is mediated by both Stat1-dependent and -independent pathways. Am J Physiol Lung Cell Mol Physiol 308: L650-L657, 2015.

35. Guo XJ and Thomas PG: New fronts emerge in the influenza cytokine storm. Semin Immunopathol 39: 541-550, 2017.

36. Holt PG, Strickland DH, Wikstrom ME and Jahnsen FL: Regulation of immunological homeostasis in the respiratory tract. Nat Rev Immunol 8: 142-152, 2008.

37. Rainer TH: L-selectin in health and disease. Resuscitation 52: 127-141, 2002.

38. Liu Q, Zhou YH and Yang ZQ: The cytokine storm of severe influenza and development of immunomodulatory therapy. Cell Mol Immunol 13: 3-10, 2016. International (CC BY-NC-ND 4.0) License. 